\title{
Prostate brachytherapy: brief history and the evolution of the different techniques
}

\author{
A. Polo*, H. Marsiglia**
}

Brachytherapy with permanent radioactive seeds is the most conformal therapy for treating localized prostate cancer. In the last 15 years there have been a renewed interest as a treatment option due to technological improvements (new ultrasound machines, better treatment planning systems, new isotopes) and the publication of encouraging clinical series from the group of investigators in Seattle.

Alexander Graham Bell was the first to describe the idea of putting radioactive sources in the prostate. In a letter dating back to the 1890's he discussed placing radium inside a glass bead and inserting it inside the prostate. However, Pasteau and Degrais in Paris reported the first actual procedures in 1913. Several other investigators followed this seminal report. This primitive technique consisted in placing non-sealed sources of radioactive gold into the prostate by an open perineal approach.

The field was significantly advanced by the work of Hilaris and Whitemore, who in the early 1970's, developed a technique for implanting the prostate with radioactive isotopes using permanent sealed sources. Using a surgical approach, they exposed the prostate gland and prepared it for seed implantation. The prostate volume was determined in the operating room and the total activity of $1-125$ required for the implant was obtained from a nomogram, specifically developed for this technique. Needles were then inserted into the prostate through the exposed retropubic space, with a free hand technique, using a finger in the rectum to guide their depth. This technique was performed over a 15 year period with what appeared to be very encouraging early results. However, it eventually became obvious that the local failure rate was higher than what would have been anticipated. In those days a failure was determined by an enlarging prostate nodule, an elevated acid phophatase or a positive bone scan. PSA was not available and post-implant prostate biopsies were not routinely done. Other problems with this technique included incorrect prostate volume measurements, loss of sources in the urinary tract, and lack of adequate dosimetric evaluation. Thus, the retropubic open implant was abandoned by the mid 1980's.

The "modern" era of prostate brachytherapy evolved from the work by Holm in Denmark who began to use transrectal ultrasound to help plan seed placement. At that time, in the early 1980's only crude axial imaging was available and very rudimentary dosimetry systems were used to plan the implant and to determine the adequacy of the procedure. Holm used a monoplanar transrectal ultrasound probe that could only image the prostate gland in transverse or axial mode. The axial images were used to construct a map for seed placement.

Ragde and Blasko extended Holm's initial work and developed an ultrasound guided transperineal technique incorporating a comprehensive planning method. Prostate images taken in a previous procedure are digitalized thanks to sophisticated software packages. These images were then reconstructed and a predictive dosimetry is generated giving a seed deposition plan. In the operating room the physician follows the plan by attempting to guide the needles with the seeds into the predetermined coordinates. Seeds can be inserted by a number of techniques. The needles can be loaded with loose seeds, before the implant (pre-loaded needles) or during the implant (Mick applicator-based technique). An alternative to loose seeds is using seeds woven in polyglycolic strands. The strands can be cut to accommodate the number of seeds required per needle.

In the recent years, progresses have been made allowing intraoperative optimization of treatment plans via dynamic dose calculations of planned needles or deposited seeds. This can open the therapeutic window improving biochemical control and reducing the associated toxicity of seed implantation.

In the corresponding session, a description of the history of permanent seed implantation in Spain will be made and a survey of the different techniques will be presented.

\footnotetext{
* Instituto Catalán de Oncología. Barcelona. España
}

** Institute Gustave Roussy. Paris. Francia 